\title{
Effect of High Voltage Impulses on Surface Discharge Characteristics of Polyethylene
}

\author{
Roya Nikjoo, Nathaniel Taylor, Hans Edin \\ School of Electrical Engineering \\ KTH Royal Institute of Technology \\ Stockholm, Sweden
}

\begin{abstract}
This paper investigates the influence of high voltage impulses on the surface discharge characteristics of polyethylene (PE) during subsequent application of 50 $\mathrm{Hz}$ AC voltage. The investigation is done on two types of PE to examine the generality of the phenomena. The effect of impulses on the surface discharge features of $\mathrm{PE}$ are studied for both cases of having either a streamer or flashover. For each case, the consecutive surface discharges behave differently when the insulation has been exposed to the transient voltages.

The effect of impulses on surface discharge of PE is shown by the change of PD numbers and phase-resolved PD patterns. The results show a prompt change in the total number of discharges which decays with the time. After a surface flashover has occurred due to the impulse, the PD behaves quite differently compared to the similar case without flashover.
\end{abstract}

\section{Introduction}

Polymeric materials have significant application in high voltage components such as cables and electrical machines and due to their good characteristics their application is increasing. Therefore, studying the electrical properties of polymers is of strong and growing interest [1]. Partial Discharges (PD) can influence the performance of electrical insulations in power components. Insulation degradation depends on the site, nature and intensity of discharges. Surface discharge on the insulation is one of the defects that can lead to a failure of the component. Knowledge of the progress of discharges and parameters that can affect them has great value on prevention of the failures. Polyethylene has a wide range of applications and specifically it is used as a major insulation of power cables and can be stressed by the power system transients.

Surface discharges that appear at the boundary of insulation are a common problem for polymers used in power components. Creation of surface discharges can increase the probability of surface breakdown.

Substantial work has been done on investigation of surface potential decay of polymers and specifically polyethylene, under corona discharge [2-6]. The effect of initial potential, charging time [5] and thickness [2] on charge decay has been investigated.

The effect of frequency and pulse polarity on surface
PD inception voltage [7], and effect of fast rising impulse on surface flashover [8] have been studied for some polymers and presented as consequences of the dielectric properties of the materials (dielectric constants, charge decay characteristics, etc.). However, behavior of PD under impulses has not been studied in such detail. Deposition, propagation and decay of charge injected by impulse voltage, and the effect on surface discharge can be of great interest to be studied. The effect of impulses can also be significant for deterioration of material and the premature aging of the insulation.

Power system transients such as lightning and switching impulses inevitably occur in power systems, and can stress the insulation of the components. Partial discharges can cause surface degradation [9]; and the severity of PD exposure can be enhanced by imposed electrical impulses. Having knowledge about the effect of impulses on the partial discharge activities of the insulation can provide valuable information for interpretation of obtained data from monitoring systems to correlate the defect and its state of development. This has a high importance for a better condition assessment of the component.

This paper investigates the influence of impulses on surface discharge characteristics of polymers. The investigations were done on several polymers; Polyethylene (PE), Polyvinyl chloride (PVC) and Polycarbonate (PC). Between them, only PE showed a consistent pattern and significant change as a result of impulses. Therefore, this paper majorly presents the results for $\mathrm{PE}$.

\section{Experimental setup}

To investigate the effect of impulses on surface discharge characteristics of PE, the IEC-b type electrode was used. The IEC-b type electrode consists of a stainless steel rod with $6 \mathrm{~mm}$ diameter and an end curvature radius of $1 \mathrm{~mm}$, and a plane circular electrode on the other side of the dielectric specimen. Two different types of PE, PE-1000 natural (UHMW) and PE-300 (HDPE), with respectively $1.5 \mathrm{~mm}$ and $1 \mathrm{~mm}$ thickness were used for the experiment. Two layers of PE were placed between the electrodes. The electric field at the air gap between the top electrode and PE surface is a function of its permittivity.

Permittivity and loss tangent of each type of PE were obtained as a function of frequency. Their dielectric responses were measured at low voltage $(200 \mathrm{~V})$ by a 
Megger IDAX 300 from $0.01 \mathrm{~Hz}$ to $10 \mathrm{kHz}$. The relative permittivity of $\mathrm{PE}$ was seen to be almost constant (2.3 for PE-300 and 2.4 for PE-1000) and the loss tangent was low (below 0.001). Frequency response of PE shows low dependency of its permittivity to the frequency which leads to its trivial change in PD inception due to the frequency [7]. A lower dielectric constant of the material leads to a lower inception voltage [7].

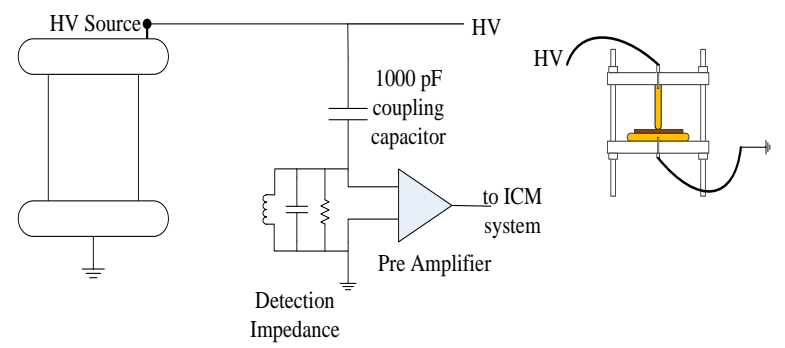

Fig. 1. Schematic of PD measurement system and test setups

The system shown in Fig. 1 was used for PD measurement. A High Voltage transformer with maximum $100 \mathrm{kV}$ output was used as the HV source.

Phase Resolved Partial Discharge (PRPD) measurements were made by an IEC60270 instrument (Power Diagnostix Gmbh, ICM system), with the detection impedance (10 $\mathrm{nF}$ capacitor, $3.9 \mathrm{mH}$ inductor and $50 \mathrm{Ohm}$ resistor all in parallel) connected in series with a $1 \mathrm{nF}$ coupling capacitor. A two-stage impulse generator was used to apply standard lightning (1.2/50 $\mu \mathrm{s})$ impulses to the test sample with voltages up to 50 $\mathrm{kV}$.

\section{Experimental procedure}

The $50 \mathrm{~Hz}$ AC voltage was applied at $6 \mathrm{kV}$ peak for PE 300 and $7 \mathrm{kV}$ for PE 1000, which was about 50\% above their PD inception voltage. PRPD patterns were recorded over 1-minute periods, separated by $30 \mathrm{~s}$ pauses. After several minutes (Fig. 2), the AC source was switched off for about 3 minutes to show how this influenced the subsequent PD without applying any impulse voltage. The AC voltage was then applied for several minutes more, after which it was turned off again and a positive standard lightning impulse with 50 $\mathrm{kV}$ amplitude was applied to the rod electrode from the impulse generator. The experiment was repeated several times with applied AC and an impulse. PRPD patterns were recorded during the intervals of $\mathrm{AC}$ voltage to see the effect of each impulse on the surface discharge parameters. The experiment was repeated on several samples; the results are shown for two samples of PE 300 (samples 1\&2) and one sample of PE 1000 (samples 3).

Charge decay is strongly dependent on temperature and moisture [3]. Therefore, the measurements were done almost at the same temperature and relative air humidity $\left(22^{\circ} \mathrm{C}\right.$ and $24 \% \mathrm{RH}$ for samples $1 \& 2,21^{\circ} \mathrm{C}$ and $20 \%$
RH for sample 3).

\section{Results and discussion}

The parameters shown in the results are the number of positive and negative discharges per cycle, obtained from the PRPD patterns of the surface discharges. Positive and negative discharges for an AC surface discharge of three PE samples are shown in Figs. 2, 5, 8. There is a prompt change in the positive discharges due to the applied impulse. By comparison with the PD observed after turning off the AC voltage but without applying an impulse voltage, this change after the impulse cannot be attributed to just the lack of AC voltage: it appears to be due to the impulse. Space charges would be injected by impulse voltage and in combination with existing space charges can change the electric field. Electric charges remain on the surface of materials for a certain time which is defined by the efficiency of the charge decay processes [10]. Charge decay can occur through several mechanisms and its time would be different for different materials and conditions.

The effect of having several impulses instead of one was investigated on some samples, but no significant differences in PD behaviors were observed. This can be explained by the negligible increase in the deposited charges on the surface, following the application of repeated impulses.

Fig.3,4 and Fig.6,7 show the changes in the PD patterns due to the impulses. An immediate increase in the number of charges can be also observed from the PD patterns.

In presence of large number of PDs, occurrence of an impulse even of low magnitude, can lead to a surface flashover and this flashover decreases the subsequent PD inception voltage. Due to the high local electric field, the density of charges close to the rod is high and by supplying the initiating charges can increase the probability of the surface breakdown. Fig. 5 and Fig. 8 also show the change of number of positive charges due to the surface flashover. However, the change and the decay are a bit different from the case of impulse.

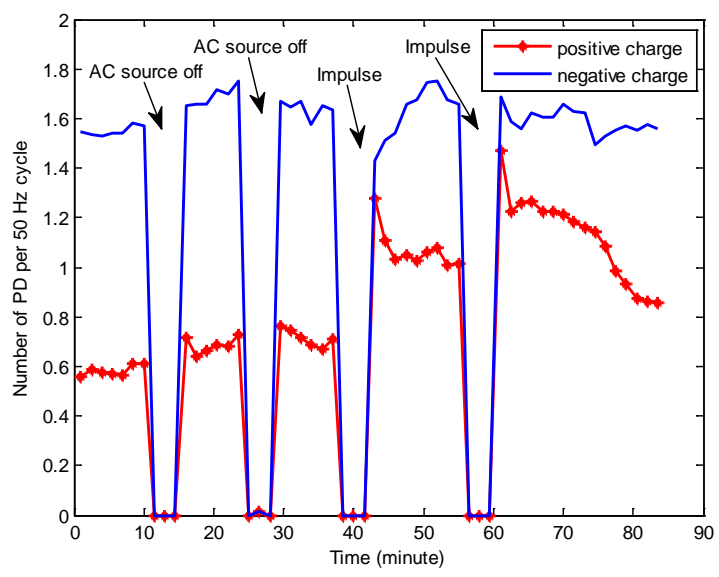

Fig. 2. Number of PD per $50 \mathrm{~Hz}$ cycle (sample 1, PE 300) 


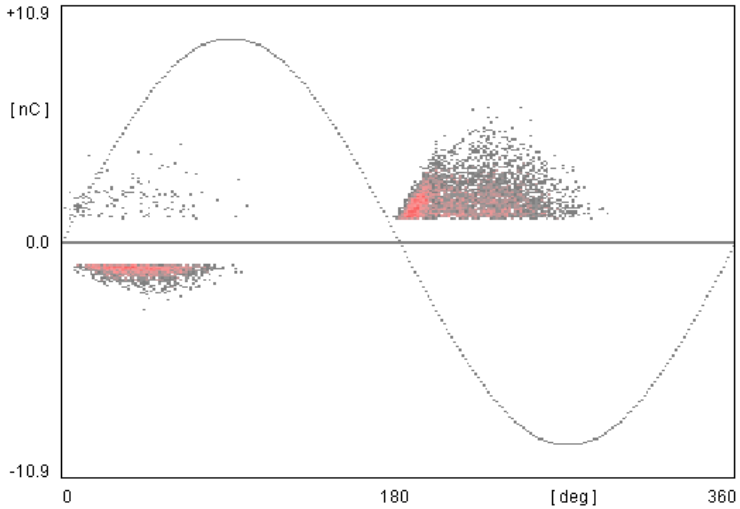

Fig. 3. PD pattern for sample 1, before HV impulse (at time 37 minutes in Figure 2)

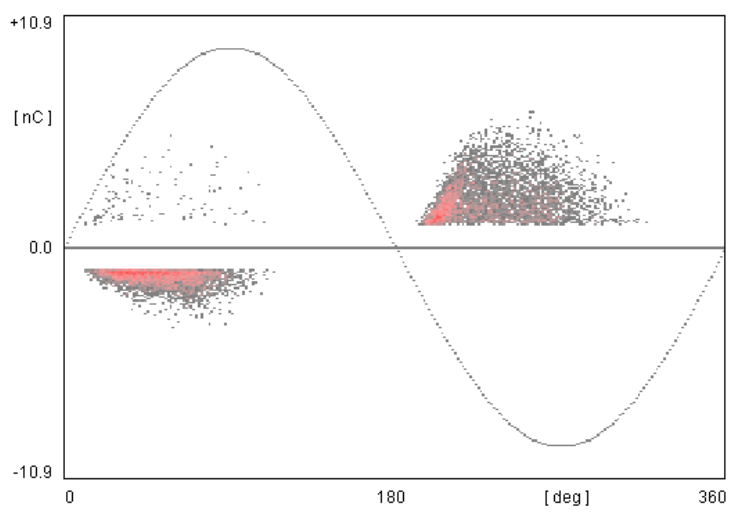

Fig. 4. PD pattern for sample 1, after HV impulse (at time 43 minutes in Figure 2)

In the case of surface breakdown a lot of charges can get neutralized and decrease the charge density (total net charge) [11] but on the other hand it can also generate ions through the gas ionization which can be adsorbed on the insulator surface and facilitate subsequent partial discharges.

It is important to consider that surface flashover is a complex mechanism and cannot be explained or formulized with few terms. It can be observed from the results that surface breakdown can lead to an increase (Fig. 5) or decrease (Fig. 8) of surface discharges. Depending on the intensity of breakdown, it can also damage the surface of the insulation.

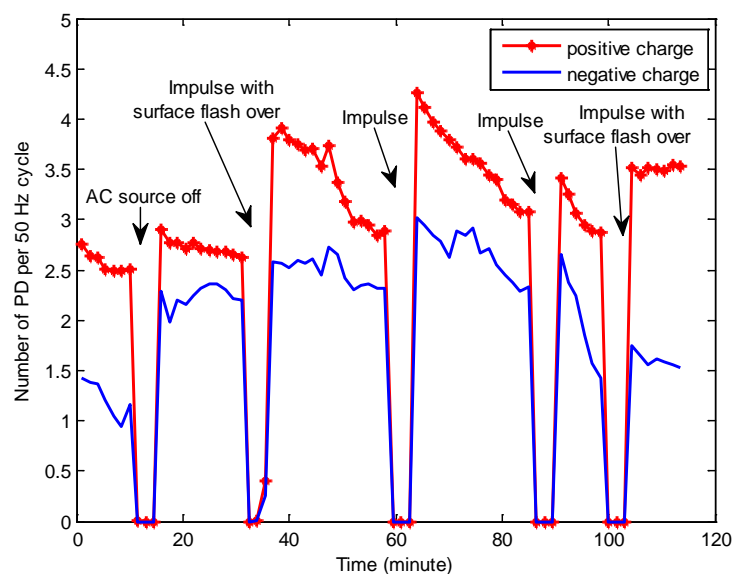

Fig. 5. Number of PD per $50 \mathrm{~Hz}$ cycle (sample 2, PE 300)

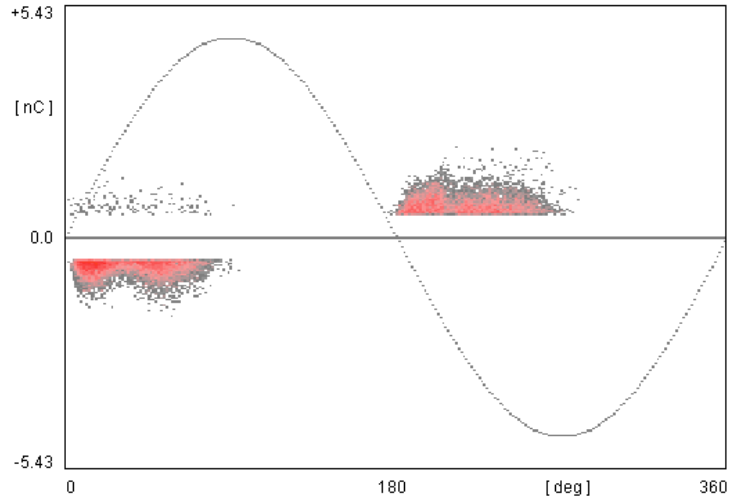

Fig. 6. PD pattern for sample 2, before $\mathrm{HV}$ impulse (at time 58 minutes in Figure 5)

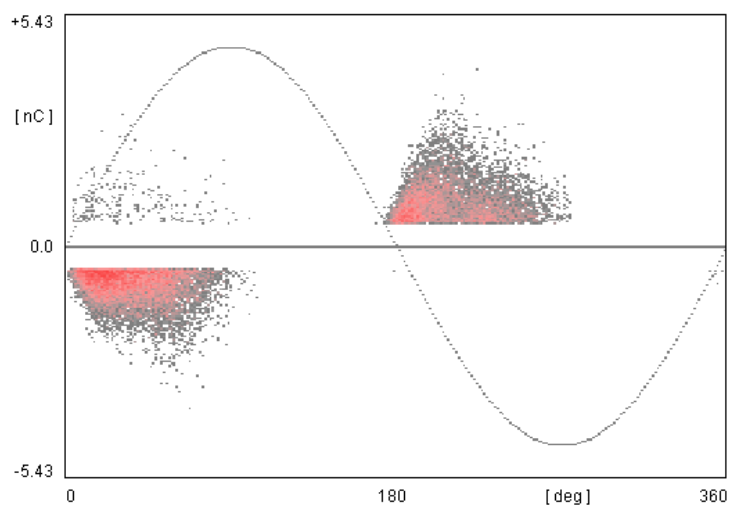

Fig. 7. PD pattern for sample 2, after HV impulse (at time 64 minutes in Figure 5)

However, the increase of positive charges due to the impulse and its decay was persistent in all samples. The charge decay can occur due to the three major mechanisms: bulk neutralization, surface conduction and neutralization by ions present in the gas phase (socalled gas neutralization).

Several parameters can generally affect the decay process of injected charges. According to [5], higher charging voltage causes faster surface charge decay in $\mathrm{PE}$ [5] due to the higher electric field. Longer charging time can also lead to a faster surface potential decay, as it allows more charge injection to the bulk and enhances surface conductivity [5].

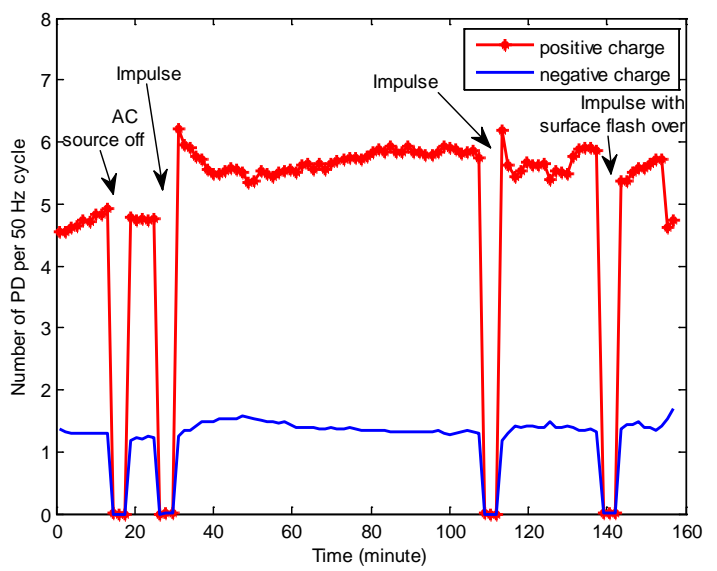

Fig. 8. Number of PD per $50 \mathrm{~Hz}$ cycle (sample 3, PE 1000) 
The material's thickness also influences the charge decay as it changes the electric field. Therefore, due to the several parameters, the complete behavior of PD including decay time constant due to the impulses can be varied from one case to another case.

Usually the abstract description of the surface charge decay can be explained in terms of ion drift, surface and bulk conduction. The ion drift (gas neurtalization) is as a function of several parameters such as ion mobility and gas (air) pressure while surface conduction is dependant on the geometry and surface conductivity [12]. However, surface conductivity itself varies with frequency and other parameters such humidity and temperature. Bulk conduction is another mechanism that is involved in charge decay and has its own contribution to the time constant of charge decay.

To compare the results obtained from polyethylene to the results from the other materials, the positive and negative discharges for PVC and PC samples with 2 $\mathrm{mm}$ thickness and using the same setup are presented in Fig. 9 and Fig. 10. From the several measurements that performed on their samples, it was interpreted that the effect of impulses on these insulations compared with $\mathrm{PE}$ is less significant and hard to be defined with a pattern due to the property of materials.

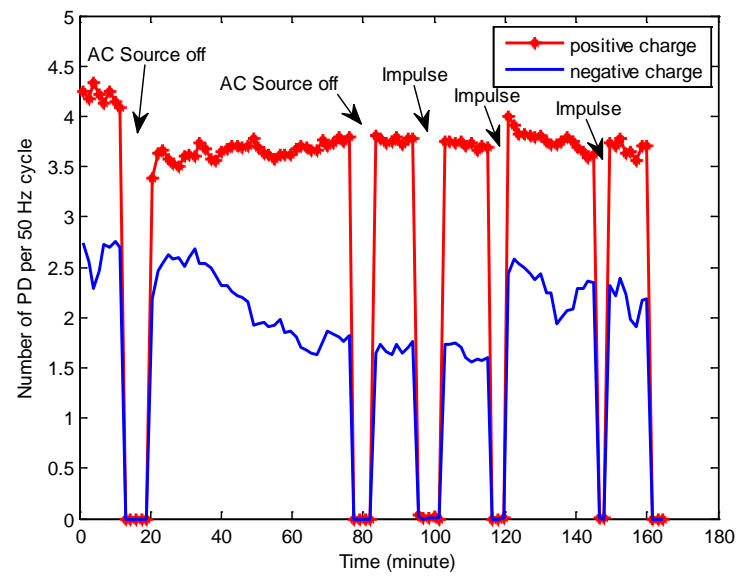

Fig. 9. Number of PD per $50 \mathrm{~Hz}$ cycle (natural PC)

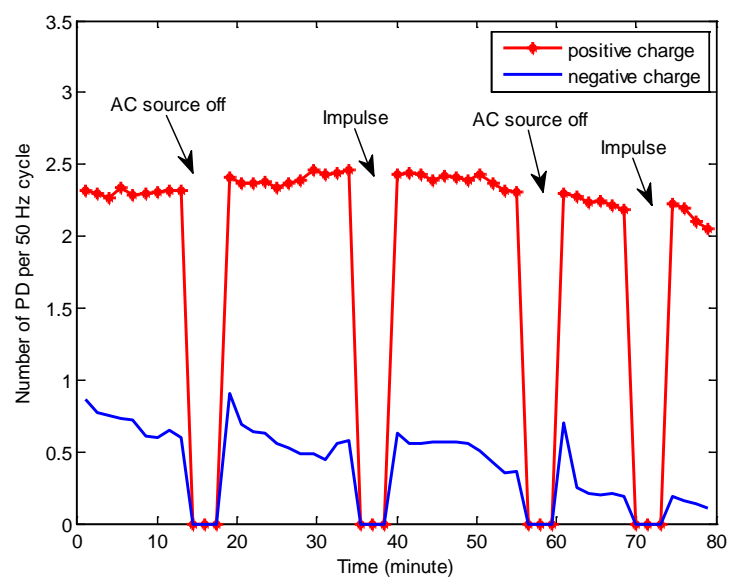

Fig. 10. Number of PD per $50 \mathrm{~Hz}$ cycle (rigid transparent PVC)

\section{Conclusion}

The effect of high voltage impulses on the surface discharge characteristics of PE is presented. This effect is shown by the prompt increase in the number of positive partial discharges due to the injection of charges, which decays thereafter. However, in the case of breakdown occurrence, the change in the PD pattern can vary for different cases: it can either increase or decrease the number of subsequent partial discharges.

The effect of voltage impulses on subsequent AC PD is of interest because of the synergistic effect of transients and $\mathrm{AC}$ PD on insulation degradation and providing useful diagnostic information.

\section{Acknowledgments}

The project is sponsored by SweGRIDS, the Swedish Centre for Smart Grids and Energy Storage. It is a partnership of academia, industry and public utilities, with major funding from the Swedish Energy Agency as well as the corporate partners.

\section{References}

[1] T. Blythe and D. Bloor, Electrical properties of polymers, Cambridge University Press, 2008.

[2] L. Zhang, Z.Xu and G.Chen, "Decay of electric charge on corona charged polyethylene”, Journal of Physics: Electrostatics, IOP Publishing Ltd, 2008.

[3] L. Herous, M.Remadnia, M. Kachi and M. Nemamcha, "Decay of Electrical Charges on Polyethylene Terephthalate Surface”, Journal of Engineering Science and Technology, Vol. 1, 2009, pp. 87-90.

[4] P. Molinié, "Measuring and modeling transient insulator response to charging: the contribution of surface potential studies", IEEE Transaction on Dielectrics and Electrical Insulation, Vol. 12, 2005, pp. 939-950.

[5] Z.Ziari, S.Sahli and A.Bellel, "Surface Potential Decay of Low Density Polyethylene (LDPE) Films under Different Corona Discharge Conditions", The Moroccan Statistical Physical and Condensed Matter Society, Vol. 12, No. 3, Dec. 2010.

[6] T. Mizutani, Y.Taniguchi and M.Ishioka, "Charge Decay and Space Charge in Corona Charged LDPE”, $11^{\text {th }}$ International Symposium on Electrets, 2002.

[7] K.Wada, K.Tsuji and H.Muto, "Partial Discharge Inception Voltage for Two Insulating Materials (PVC and PE) under Inverter Surge Voltage”, 7th International Conference on Properties and Application of Dielectric Materials, Nagoya, June 2003.

[8] M.P.Wilson, M.J.Given, I.V.Timoshkin, S.J.MacGregor, M.A.Sinclair and K.J.Thomas, "Impulse-Driven Surface Flashover of Polymeric 
Dielectric Materials Immersed in Insulating Oil”, 44th International Universities Power Engineering Conference (UPEC), Glasgow, September 2009.

[9] M.Kozako, N.Fuse, Y.Ohki, T.Okamoto and T.Tanaka, "Surface Degradation of Polyamide Nanocomposites Caused by Partial Discharges Using IEC (b) Electrodes", IEEE Transaction on Dielectrics and Electrical Insulation, Vol. 11, No. 5, June 2004, pp. 833-839.

[10] Sarath Kumara, "Electrical Charges on Polymeric Insulator Surfaces and their Impact on Flashover Performance," Ph.D. thesis. Department of Materials and Manufacturing Technology, Chalmers university of technology, Gothenburg, Sweden 2012.

[11] N.L. Allen and D.C. Faircloth, "Corona Propagation and Charge Deposition on PTFE surface”, IEEE Transaction on Dielectrics and Electrical Insulation, Vol. 10, No. 2, April 2003, pp. 295-304.

[12] L.Niemeyer, "A Generalized Approach to Partial Discharge Modeling”, IEEE Transaction on Dielectrics and Electrical Insulation, Vol. 9, No. 4, August 1995, pp. 510-528. 